\title{
Musique et plasticité cérébrale
}

Music and brain plasticity

Música y plasticidad cerebral

\section{Laura Ferreri}

Traducteur : Jane-Mary Rivière

\section{(2) OpenEdition}

1 Journals

Édition électronique

URL : https://journals.openedition.org/ries/5944

DOI : 10.4000/ries.5944

ISSN : 2261-4265

Éditeur

France Education international

\section{Édition imprimée}

Date de publication : 1 septembre 2017

Pagination : 55-63

ISBN : 978-2-85420-615-9

ISSN : $1254-4590$

\section{Référence électronique}

Laura Ferreri, « Musique et plasticité cérébrale », Revue internationale d'éducation de Sèvres [En ligne], 75 | septembre 2017, mis en ligne le 01 septembre 2019, consulté le 24 juin 2021. URL : http:// journals.openedition.org/ries/5944; DOI : https://doi.org/10.4000/ries.5944 


\title{
Musique et plasticité cérébrale*
}

\author{
Laura Ferreri \\ Université de Barcelone / Institut biomédical \\ IDIBELL, Barcelone
}

\section{L'INTÉRÊT NEUROSCIENTIFIQUE POUR LA MUSIQUE}

La musique a une présence constante et unique tout au long de la vie humaine. À travers les siècles, de nombreux philosophes, artistes et scientifiques ont tenté de rendre pleinement compte du rôle que la musique joue dans nos vies en montrant comment elle peut influencer nos émotions, notre cognition et notre comportement. Durant les deux dernières décennies, les sciences du cerveau ont porté une attention sans précédent à la musique, de plus en plus considérée comme "la nourriture des neurosciences"(Zatorre, 2005). Quelles caractéristiques particulières de la musique intéressent-elles tant la communauté neuroscientifique?

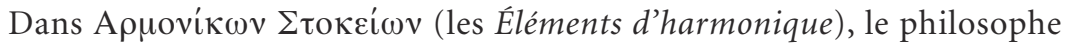
Aristoxène (Tarente, fin du IV siècle av. J.C.) est le premier philosophe grec à avoir affirmé que comprendre une composition musicale signifie suivre le processus de sa mélodie avec l'oreille et l'intellect. L'oreille détecte les amplitudes de la mélodie qui se succèdent, alors que l'intellect envisage les fonctions des notes à l'intérieur du système auquel elles appartiennent. Plus précisément, Aristoxène propose deux procédés principaux impliqués dans l'écoute de la musique : la mémoire, pour retenir le passé, et les sens, à savoir la perception, afin d'appréhender le présent. Cette conception intuitive, qui contrastait avec l'approche mathématique de la musique par Pythagore, était extrêmement novatrice, à la lumière des récentes découvertes neuroscientifiques.

En effet, l'intérêt neuroscientifique pour la musique n'est pas simplement motivé par la possibilité fascinante de trouver les corrélations neurales de ce langage sensoriel basé sur des règles, temporellement ordonné et extrêmement complexe. Dans Musicophilia, Oliver Sacks (2007) affirme que « les humains sont une espèce musicale autant que linguistique ». À peu d'exceptions près, tout le monde peut percevoir la musique, les tonalités, le timbre, les intervalles de hauteur tonale ainsi que le rythme, les courbes mélodiques et l'harmonie. Toutes ces caractéristiques sont ensuite assemblées (la plupart du temps implicitement)

* Article traduit de l’anglais par Jane-Mary Rivière. 
à l'aide de différentes parties du cerveau et ceci est généralement accompagnée d'une réaction émotionnelle intense et profonde (Koelsch, 2014). Par conséquent, le véritable défi porté par la musique dans le domaine neuroscientifique commence à partir de preuves que la musique, en tant que stimulus auditif très complexe avec un fort impact émotionnel, implique tout le cerveau à travers un ensemble diversifié d'opérations perceptives et cognitives et donc des substrats neuronaux également divers. Comme l'étude de la physiologie et de la neurologie cérébrales est fondamentale pour comprendre le fonctionnement du cerveau, la musique est devenue un modèle très utile pour la recherche sur le cerveau relative à la perception et à la cognition, en mesure d'apporter des contributions intéressantes aux connaissances générales sur le cerveau : comment il fonctionne et comment il peut modifier sa structure et ses fonctions en réaction à la stimulation externe.

\section{Qu'est-CE LA PLASTICITÉ CÉRÉBRALE ?}

On entend par plasticité cérébrale la capacité du cerveau à modifier sa structure, sa fonction et sa connectivité. Une augmentation de la plasticité cérébrale est associée à des changements multiples et dissociables du cerveau en corrélation avec des résultats comportementaux, y compris des augmentations de la longueur dendritique, des augmentations ou des diminutions dans la densité de la colonne vertébrale, la formation de synapses, une activité gliale accrue et une activité métabolique altérée. Qui plus est, ces changements peuvent se produire tout au long de la vie d'un individu et ne sont pas limités à une fenêtre critique pendant les premières années de la vie, comme on le pensait auparavant.

Il y a près de vingt ans, un groupe de chercheurs du Royaume-Uni a mené une étude très connue sur les chauffeurs de taxi londoniens, qui avaient des compétences en navigation particulièrement développées. Après avoir scanné leur cerveau par le biais de l'imagerie par résonance magnétique (IRM), Maguire et ses collaborateurs (2000) ont constaté que les chauffeurs de taxi avaient un hippocampe plus volumineux, l'hippocampe étant une région du cerveau spécifiquement impliquée dans la formation de la mémoire, par rapport aux sujets témoins qui avaient une mémoire spatiale moins développée. De manière significative, cette étude a non seulement confirmé le rôle de l'hippocampe dans les représentations spatiales, mais a également indiqué une capacité pour des variations locales de la structure du cerveau sain d'un humain adulte en réponse aux exigences environnementales. Nous savons maintenant que ces changements se produisent également après des formations courtes et que seulement quarantecinq minutes d'entraînement sur une tâche de navigation sur un jeu vidéo semblent suffire pour modifier la structure et la connectivité de l'hippocampe. 
La capacité d'induire la plasticité cérébrale est strictement lié à une perspective de remédiation, comme la nécessité d'une récupération fonctionnelle suite à des lésions cérébrales. En effet, le système nerveux central est capable de récupérer et d'adapter des mécanismes de compensation secondaires aux lésions ( $\mathrm{Su}$ et al., 2016). En faisant de la remédiation pour réapprendre les tâches de base, un patient souffrant d'une lésion cérébrale traumatique peut être capable de former de nouvelles connexions cérébrales lui permettant de récupérer les capacités perdues. Plusieurs études ont donc essayé de stimuler la plasticité cérébrale en proposant des stratégies de remédiation visant à atténuer les déficits associés à des pathologies spécifiques, tels que les déficits physiques après des accidents vasculaires cérébraux ou des pathologies focales provoquant l'aphasie. Par conséquent, il est extrêmement important de comprendre ce qui peut stimuler le cerveau spécifiquement et donc modifier sa plasticité et améliorer les résultats comportementaux.

Dans ce cadre, la musique, capable de stimuler le cerveau entier, apparaît comme étant un stimulus unique. Plusieurs auteurs ont étudié les effets de la pratique et de l'écoute musicales sur les fonctions cognitives et neurales. Les données tant comportementales que de neuro-imagerie ont révélé l'ampleur et la nature plastique de la réaction du cerveau à la stimulation musicale. Dans cet article, je développerai donc deux principaux thèmes liés à la plasticité cérébrale produite par la musique : tout d'abord, l'effet de la formation musicale sur le cerveau ; ensuite, l'utilisation de la musique pour stimuler et réhabiliter les fonctions cognitives.

\section{MUSIQUE ET PLASTICITÉ CÉRÉBRALE \\ La formation musicale et le cerveau}

Si la musique peut stimuler et changer l'organisation neurale, le cerveau des musiciens est-il différent de celui des non musiciens? Plusieurs expériences sur l'expertise musicale ont grandement contribué à comprendre l'immense gamme des changements de plasticité liés à la musique.

Ouvrant les portes à la recherche sur la plasticité musicale, Ramon et Cajal affirment (voir Ramon, Cajal, Pasik et Pasik, 1999) que :

tout le monde sait que la capacité d'un pianiste [de jouer] en s'adaptant à la nouvelle œuvre demande de nombreuses années de gymnastique mentale et musculaire. Pour comprendre ce phénomène important, il est nécessaire d'admettre que, parallèlement au renforcement des voies organiques préétablies, de nouvelles voies d'accès sont créées par la ramification et la croissance progressive des processus dendritique et axonal terminaux.

Jouer un instrument de musique est en effet une expérience intense, multi-sensorielle, motrice, cognitive et émotionnelle qui commence généralement à un âge précoce et exige l'acquisition et le maintien d'un ensemble de 
compétences au cours de la vie d'un musicien. La raison en est qu'une pratique musicale constante est capable de modifier l'organisation du cerveau en augmentant le nombre de neurones employés pour un certain processus, soutenant leur synchronisation temporelle et augmentant le nombre et la force des connexions synaptiques excitatrices et inhibitrices.

Plusieurs études par neuro-imagerie ont démontré que la pratique musicale est capable de modifier l'organisation du cerveau dans les cortex auditifs, somatosensoriel et moteur, chez les enfants et les adultes. En particulier, ces régions montrent généralement une augmentation du volume ou de la densité de la matière grise et une amélioration de l'organisation microstructurale de la matière blanche chez les musiciens, par rapport aux individus sans formation musicale.

Il est important de noter que la plupart des études sur l'expertise musicale se concentrent sur la formation musicale précoce. En effet, bien que la plasticité cérébrale soit un phénomène qui concerne la durée de vie entière, le système nerveux central présente sa malléabilité maximale durant les premières phases du développement. Pour cette raison, la formation musicale précoce, qui commence au cours de l'enfance et l'adolescence, peut avoir un bénéfice et un effet maximum sur le cerveau. Toutefois, des résultats cruciaux ont également été obtenus avec des débuts tardifs et une formation musicale courte. Par exemple, des adultes non musiciens qui ont appris à jouer une séquence à cinq doigts sur un clavier en seulement cinq jours ou à jouer au piano et à lire une partition musicale en quinze semaines ont montré une meilleure performance comportementale accompagnée d'une réorganisation du cerveau cortical (voir Dalla Bella, 2016, pour une revue).

Il est intéressant de noter que l'expertise musicale peut aussi influer sur la réorganisation des régions corticales et sous-corticales essentielles pour d'autres fonctions cognitives non musicales, telles que les processus de langage, les fonctions exécutives ou les processus de mémoire. Conformément à cela, des études récentes sur le vieillissement suggèrent que les changements du cerveau liés à la pratique musicale augmentent la résistance au vieillissement cognitif. Par exemple, une étude récente sur des cojumeaux a révélé que les jumeaux qui jouent un instrument de musique étaient moins susceptibles de développer une démence et une déficience cognitive que leurs cojumeaux non musiciens (Balbag et al., 2014). Ensemble, toutes ces études suggèrent que les changements neuroplastiques liés à la formation musicale peuvent se produire rapidement, à différents stades de la vie, et peuvent avoir des conséquences comportementales importantes sur les capacités non musicales également.

Si la formation musicale est capable de stimuler et d'induire la plasticité cérébrale facilement dans les régions impliquées dans des capacités non musicales également, il est raisonnable d'émettre l'hypothèse que non seulement la formation musicale mais aussi la simple exposition à la musique pourraient moduler les réactions cérébrales et influencer positivement les fonctions non 
musicales aux niveaux à la fois comportemental et fonctionnel. La section suivante se concentrera donc sur l'effet de l'exposition à la musique sur la stimulation d'autres fonctions cognitives, ouvrant ainsi des perspectives importantes pour les paradigmes de remédiation.

\section{De la plasticité cérébrale à la stimulation et à la remédiation cognitives}

Un grand nombre d'études ont révélé la façon dont la musique peut améliorer les capacités non musicales de fonctions cognitives plus perceptuelles à des fonctions cognitives supérieures et plus générales en réaction non seulement à la formation musicale, mais aussi à une simple exposition musicale. Ici, je me concentrerai sur trois processus principaux : la musique, le langage et la mémoire, afin de présenter la façon dont les changements du cerveau liés à la musique peuvent moduler la cognition et le comportement humains.

\section{Mouvement}

Un lien intrinsèque existe entre la musique et le mouvement: nous n'avons pas besoin d'être des musiciens experts pour commencer à frapper des mains, taper des pieds ou danser quand une pièce musicale commence. Au contraire, parfois, nous ne pouvons tout simplement pas nous empêcher de bouger notre corps et de suivre le rythme d'un extrait musical que nous aimons. Dans le domaine de la recherche sur la musique et les mouvements, la plupart des études ont mis l'accent sur la composante rythmique et sur la capacité des humains à se synchroniser avec une stimulation musicale donnée. Par exemple, il a été démontré que la musique, via la capacité de synchronisation avec un rythme auditif donné, peut influencer la manière et la fréquence à laquelle les sujets marchent. Au niveau neurophysiologique, un tel phénomène est connu sous le nom d'entraînement neuronal et fait référence au fait que nos oscillations neuronales internes sont capables de modifier leur fréquence pour se synchroniser sur des oscillations (musicales) externes. Cet effet de la musique sur le cerveau et le mouvement pourrait-il améliorer les fonctions motrices altérées? En 1996, Thaut et al. ont suggéré que la stimulation auditive rythmique pourrait être utilisée pour atténuer les déficits moteurs de la maladie de Parkinson. Ils ont comparé un groupe de patients atteints de la maladie de Parkinson suivant un programme d'entraînement à la marche à domicile basé sur une stimulation auditive rythmique avec un groupe témoin qui avait participé à un programme d'entraînement interne à son propre rythme. Après trois semaines, les patients qui s'étaient exercés avec la stimulation auditive rythmique, par rapport au groupe témoin, avaient amélioré de manière significative la vitesse de leur démarche, leur longueur de foulée et la cadence de leurs pas. Un autre axe de recherche clinique concerne la rééducation suite à un AVC, durant laquelle trois 
semaines de thérapie soutenue par de la musique avec un piano MIDI $^{1}$ ou une batterie électronique ont produit des améliorations significatives des compétences globales fines associées à des changements neuraux spécifiques indiquant une meilleure connectivité corticale et une activation améliorée du cortex moteur (Altenmüller et al., 2009).

\section{Langue}

Comme Tillmann (2012) l'a évalué, la recherche en matière de cognition musicale éclaire non seulement la compréhension des processus musicaux, et linguistiques ainsi que celle d'autres stimulus structurés. En effet, la musique et le langage partagent plusieurs ressources neuronales, ce dont il est possible d'apporter la preuve tant au niveau perceptif que cognitif. Par exemple, grâce aux propriétés structurantes de la musique qui pourraient soutenir l'organisation linguistique et améliorer sa compréhension, il est plus facile d'apprendre des séquences chantées que des paroles. De même, les propriétés syntaxiques d'une structure musicale constituent un lien direct avec la langue. Dans une étude bien connue, Patel et al. (1998) ont testé la spécificité linguistique du P600, une corrélation du potentiel du cerveau lié aux événements, au traitement syntaxique. En comparant les incongruités syntaxiques dans le langage et la musique, les résultats ont révélé que les incongruités structurelles linguistiques et musicales suscitaient des incongruités statistiquement indiscernables. Ces liens bien établis entre la musique et le langage expliqueraient un effet musical positif sur les processus linguistiques non musicaux. Selon Patel (2011), cinq conditions peuvent expliquer la façon dont la musique profite au codage neuronal de la parole (l'hypothèse OPERA) : (1) le chevauchement dans les réseaux du cerveau qui traitent une caractéristique acoustique utilisée dans la musique et le discours ; (2) le fait que, en termes de précision de traitement, la musique impose des exigences plus élevées sur ces discours partagés ; (3) les fortes émotions positives suscitées par la musique; (4) la répétition donnée par une formation musicale et (5) l'attention accrue qu'entraîne la musique.

Quant au mouvement, les caractéristiques rythmiques et mélodiques des stimuli musicaux sont utilisées dans le domaine clinique pour améliorer la performance linguistique. Un bon exemple est donné par la Melodic Intonation Therapy (MIT), un processus thérapeutique utilisé par les musicothérapeutes et les orthophonistes pour aider les patients atteints de troubles de la communication causés par des lésions cérébrales. Le MIT est basé sur les observations selon lesquelles certains patients aphasiques sont aidés dans la parole s’ils doivent produire des mots chantés plutôt que dits et si le rythme est frappé avec les mains. On a démontré que la musique améliore la performance linguistique également chez les enfants dyslexiques, pour lesquels une formation musicale

1. La norme MIDI (Musical Instrument Digital Interface - Interface numérique pour instruments de musique) est un protocole de communication standard qui permet de faire transiter des informations musicales d'un instrument à l'autre ou d'un ordinateur à un instrument et réciproquement. (NdIR) 
peut améliorer considérablement leurs capacités de frapper le rythme (c'est-àdire leur capacité à reproduire des structures rythmiques) ainsi que des traitements auditifs et les compétences phonologiques (voir par exemple Flaugnacco et al., 2014). Dans ce cas, la valence émotionnelle positive et la synchronisation rythmique produites par la stimulation musicale peuvent expliquer l'amélioration du langage par la musique.

\section{Mémoire}

Combien d'entre nous peuvent se souvenir facilement des paroles d'une chanson, mais se sentent totalement incapables d'apprendre un poème par cour? Pourquoi avons-nous le sentiment que l'on peut mieux se souvenir d'une information difficile à encoder si elle est chantée, plutôt que dite ? La recherche neuro-cognitive a effectué des études très poussées sur les mécanismes qui pourraient servir de médiateur à cette facilitation mnémonique musicale (voir Ferreri et Verga, 2016). Il a été proposé que la musique peut aider à l'encodage des sujets en raison de ses caractéristiques perceptuelles intrinsèques : la structure rythmique ainsi que l'organisation séquentielle des phrases musicales et des variations de mélodies peuvent jouer un rôle crucial dans la formation de la mémoire, par exemple en attirant l'attention des sujets sur les informations pertinentes à retenir ou en permettant le traitement de la manipulation profonde. En outre, il est important de souligner le pouvoir émotionnel et évocateur de la musique : un extrait musical peut être profondément évocateur en nous transportant dans sa "beauté pure » et évoquant ainsi des émotions spécifiques et des souvenirs d'événements, de rencontres ou d'états d'esprit qui ne peuvent être évoqués d'une autre manière.

Cela devient particulièrement pertinent dans le domaine clinique, où l'idée qu'un extrait musical pourrait évoquer des expériences de vie particulières et des détails épisodiques connexes possibles a conduit plusieurs auteurs à enquêter sur la relation entre la musique et la mémoire, en particulier chez les patients atteints de la maladie d'Alzheimer. Par exemple, on a montré que la musique peut, à la fois, améliorer sensiblement les patients atteints d'une forme modérée de la maladie d'Alzheimer à se souvenir d'épisodes autobiographiques et réduire les niveaux d'anxiété. Par exemple, El Haj et al. (2012) ont testé les mémoires autobiographiques de patients modérément atteints d'Alzheimer en utilisant une méthode narrative libre sous condition "silence ", sous condition "musique " dans laquelle des sujets étaient exposés aux Quatre saisons de Vivaldi, et sous condition "musique choisie ", dans laquelle des sujets étaient exposés à la musique de leur choix. Les résultats ont indiqué une meilleure performance sous les conditions «Quatre saisons» et "choisie », chacune étant plus élevée que dans la condition «silence ». En outre, observant que l'amélioration autobiographique a été également caractérisée par un perfectionnement des mots émotifs positifs, les auteurs ont conclu que la musique améliore la performance de la mémoire autobiographique en favorisant des mémoires émotives positives. 
Ensemble, les études analysées dans le domaine du langage, du mouvement et de la mémoire suggèrent que la musique est un bon outil de stimulation cognitive, capable de moduler la plasticité cérébrale et de stimuler un large éventail de performances cognitives.

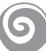

Le cerveau humain a la capacité inouïe - la dénommée plasticité cérébrale - de changer sa structure et sa fonction en réponse à une stimulation externe tout au long de la vie d'un individu. La musique est une présence unique tout au long de notre vie et constitue un stimulant riche et complexe capable de stimuler tout le cerveau. L'apprentissage et la pratique de la musique sont des activités multimodales impliquant des processus perceptifs, moteurs, sensoriels, émotionnels et cognitifs associés à des réseaux neuronaux complexes : plusieurs études sur les enfants et les adultes ont montré que la formation musicale courte et longue est capable de façonner la structure et les fonctions du cerveau, induisant ainsi des processus de plasticité cérébrale. En outre, pratiquer et écouter de la musique chez les non musiciens s'est avéré fortement capable de moduler l'activité cérébrale. Dans ce cadre, plusieurs études ont souligné que la capacité de la musique à stimuler et à modifier les fonctions du cerveau peut être employée pour améliorer d'autres capacités non musicales telles que le mouvement, le langage ou la mémoire, avec des conséquences importantes pour les interventions neuro-réhabilitatives.

\section{BibLIOgRAPHIE}

ALTENMÜLLER E. O., MARCO-PALLARES J., MÜNTE T. F., SCHNEIDER S. (2009) : "Neural reorganization underlies improvement in stroke-induced motor dysfunction by music supported therapy ", Annals of the New York Academy of Sciences, 1169, 395-405.

BALBAG M. A., PEDERSEN N. L., GATZ M. (2014) : «Playing a musical instrument as a protective factor against dementia and cognitive impairment: a population-based twin study ", International Journal of Alzheimer's Disease.

CAJAL S. R. y., PASIK P., PASIK T., KARTEN H. J. (1999) : "Texture of the Nervous System of Man and the Vertebrates: Vol I », Nature, 402(6759), 235-235.

DALLA BELLA S. (2016) : "Music and brain plasticity", The Oxford handbook of music psychology, 325.

EL HAJ M., POSTAL V., ALLAIN P. (2012) : "Music enhances autobiographical memory in mild Alzheimer's disease, Educational Gerontology, 38(1), 30-41.

FERRERI L., VERGA L. (2016) : Benefits of Music on Verbal Learning and Memory. Music Perception: An Interdisciplinary Journal, 34(2), 167-182.

FLAUGNACCO E., LOPEZ L., TERRIBILI C., ZOIA S., BUDA S., TILLI S., SCHÖN D. (2014) : "Rhythm perception and production predict reading abilities in developmental dyslexia », Frontiers in human neuroscience, 8. 
MAGUIRE E. A., GADIAN D. G., JOHNSRUDE I. S., GOOD C. D., ASHBURNER J., FRACKOWIAK R. S., FRITH C. D. (2000) : « Navigation-related structural change in the hippocampi of taxi drivers ", Proceedings of the National Academy of Sciences, 97(8), 4398-4403.

PATEL A. D. (2011) : "Why would musical training benefit the neural encoding of speech? The OPERA hypothesis ", Frontiers in Psychology, 2.

SACKS O. (2007) : Musicophilia, New York: Alfred A.

THAUT M. H., MCINTOSH G. C., RICE R. R., MilleR R. A., RATHbUN J., BRAULT J. M. (1996) : Rhythmic auditory stimulation in gait training for Parkinson's disease patients. Movement disorders, 11(2), 193-200.

TILLMANN B. (2012) : "Music and language perception: expectations, structural integration, and cognitive sequencing ", Topics in cognitive science, 4(4), 568-584. 
